\title{
Optimising Geriatric Care in the COVID Era
}

Senthil Meenrajan, $M D^{1^{*}}$ and Dianne Goede, $M D^{2}$

${ }^{1}$ Associate Professor of Medicine, University of Florida, FL, USA

${ }^{2}$ Assistant Professor of Medicine, University of Florida, FL, USA

A combination of complex physical, cognitive, functional and social issues in the geriatric patient make evaluation and management uniquely challenging. Undoubtably, the COVID 19 pandemic has complicated the medical care of the geriatric patient even further. Physicians are challenged to safely meet the unique needs of this patient population while also ensuring they best mitigate the risk of COVID-19 exposure and infection. Even prior to the COVID-19 pandemic the geriatric patient faced a number of unique problems. Younger adults typically visit their primary care physician (PCP) annually and for acute medical problems as they have relatively few chronic medical illnesses. The older patient however often has a multitude of chronic medical problems, with periodic exacerbations. Management requires coordination of several visits to various consultants/subspecialists in addition to their PCP. Making it to all the appointments poses a logistical challenge, considering the combination of medical and functional problems. Beyond physical debility and limitations, cognitive decline also interferes with care of the geriatric patient including: Missed appointments, inability to follow medication regimen correctly, risk for safety issues including driving, fire, money management etc. Use of multiple medications and the adverse effects of polypharmacy is another unique challenge in this group. A combination of social, nutritional and functional problems can each contribute to further complicate care.

The COVID-19 pandemic has impacted the lives of everyone and drastically affected the way healthcare is delivered. As the elderly are typically more medically complex and are seen by multiple physicians, the impact of these changes on this population is magnified. Lock down, quarantine, social distancing and other restrictions impacts the daily lives of the older population in many challenging ways. Given their higher risk of morbidity and mortality from COVID-19 infection [1], many of these patients choose to stay home. As public transportation options have decreased, it translates to decreased access to food, medications, social interactions and medical care. Less interaction with friends, family and neighbours may result in causing or worsening depression, especially worrisome for a patient population already prone for this problem from other risk factors [2]. Increased time at home in isolation can also worsen substance abuse problems especially alcohol. Even if the patient recognises some of these problems, access to care can be difficult as some physician offic- es have restricted access. Furthermore, many of the elderly are apprehensive of visits to clinics, hospitals and emergency rooms due to fear of contracting COVID infection. These factors all compromise the health of these older patients and the longer the pandemic drags on, the higher the risk of worsening chronic medical problems, physical deconditioning and mental health distress.

Many of these concerns and gaps in the care of the elderly are not new; just exacerbated now. In an attempt to help mitigate COVID spread to our highest risk patients and bridge gaps in healthcare delivery, Telemedicine has grabbed a more prominent role in healthcare delivery in the last few months. Many practices offered some or all of their services through telemedicine visits. In the US and some other countries, improved reimbursement for telemedicine visits has resulted in faster and more widespread adoption of this modality of care delivery. In the younger patient, with relatively few medical concerns, this technology may lend itself well to address the healthcare needs of the individual. However, in the older patient with a number of acute and chronic concerns that need to be addressed, telemedicine can be a solution and a problem.

Telemedicine offers a safe modality of evaluation and management to elderly patients [3]. Patients who are concerned about the risks of COVID exposure by visiting a healthcare facility are likely to cancel appointments or otherwise 'no show'. Telemedicine visits are a convenient option to keep the patient in their home while they receive needed evaluation and management. Telemedicine visits also offer other benefits in the care of the older patient beyond safety and convenience:

- Obviate the need for transportation or for someone to bring these patients to appointments.

*Corresponding author: Senthil Meenrajan, MD, Associate Professor of Medicine, University of Florida, 2000, Internal Medicine, SW Archer Road, Gainesville, 32606, FL, USA, Tel: +1-(352)$672-8401$

Accepted: March 01, 2021

Published online: March 03, 2021

Citation: Meenrajan S, Goede D (2021) Optimising Geriatric Care in the COVID Era. T Geriatr Med 3(1):55-56 
- Ability to easily attend multiple appointments with different physicians on the same day.

- Ability to do a 'brown bag review' to reconcile medications for the patient and to avoid duplications, omissions or other medication errors. This type of inventory check can be accomplished more reliably with the patient at home.

- A better understanding of the patient's home and living environment through visual video connectivity.

- Potential for family members, who may be at work or other locations, to join in the telemedicine visit.

Telemedicine is not a new or radical concept in geriatric care. A number of pilot and feasibility studies have gone on for more than a decade. In 2015 a review study reported that $31 \%$ of older patients had high satisfaction with telemedicine visits and another $31 \%$ had high acceptance rate [4]. As the technology has advanced in the last few years, ease of use and improved functionality make it easy to adopt. The hardware and software requirements are less now and oftentimes a personal smartphone is all that is required.

All benefits notwithstanding, telemedicine for geriatric care is not perfect either. Despite the common notion that everyone over 65 years is geriatric, there is great heterogeneity in that group. People in their 60s and probably even early 70s have lived with, used and often worked with computers during their careers. Most of them probably also have adequate exposure to smart phone technology and different apps over the last 10 years. The same cannot be said of people in their 80s; many probably never used computers or smartphones and are not tech savvy to use telemedicine platforms comfortably. Beyond this, additional challenges with telemedicine include:

- Patients may have cognitive, vision and hearing problems that interfere with their ability to use these technologies effectively. Earlier studies have reported significant technical challenges in the widespread implementation of telemedicine visits in this population.

- Concern about potential inappropriate use of telemedicine visits for symptoms which warrant clinical examination. Lost opportunity to pick up early findings of disease like crackles on lung exam or new skin lesion may be lost.
- Patients may postpone periodic blood tests to follow up on things like renal function, $\mathrm{HbA1C}$ etc. These tests are typically done at the time of office visits and telemedicine visits may give the false sense of 'everything's been looked into'.

- Lastly, since family members may be with patients during telemedicine visits and the space at home can be limited, there is a concern for lack of privacy for older patients to have candid conversations with their physicians about problems related to sexual health, abuse or other personal topics.

In summary, geriatric care presents unique challenges. The COVID pandemic, by virtue of the additional disease morbidity and mortality risks posed to elderly and by forcing changes in healthcare delivery, brings many of these concerns in geriatric care to the spotlight. Telemedicine has great potential to alleviate some of these problems but also comes with its own set of limitations. Successful care delivery will require thoughtful, judicious use of technology with careful assessment of the patient's capabilities, current needs and long term goals.

\section{Conflict of Interest}

None.

\section{Funding}

None.

\section{References}

1. https://www.cdc.gov/coronavirus/2019-ncov/need-extra-precautions/older-adults.html

2. Fiske A, Wetherell JL, Gatz M (2009) Depression in older adults. Annu Rev Clin Psychol 5: 363-389.

3. Merrell RC (2015) Geriatric Telemedicine: Background and Evidence for Telemedicine as a Way to Address the Challenges of Geriatrics. Healthc Inform Res 21: 223-229.

4. Narasimha S, Madathil KC, Agnisarman S, et al. (2017) Designing Telemedicine Systems for Geriatric Patients: A Review of the Usability Studies. Telemed J E Health 23: 459-472.

DOI: $10.36959 / 452 / 583$ 